\title{
Recombinant growth hormone treatment, osteoporosis and fractures, more complicated than it seems!
}

\author{
Maria Fleseriu ${ }^{1}$
}

Received: 11 December 2017 / Accepted: 29 December 2017 / Published online: 19 January 2018

(c) Springer Science+Business Media, LLC, part of Springer Nature 2018

Growth hormone (GH) effects on skeletal development are well recognized. $\mathrm{GH}$ promotes longitudinal bone growth during childhood and attainment of adult bone mass [1, 2]. However, GH also plays a role in adult skeletal mass maintenance. Insulin-like growth factor 1 (IGF-1) influences development and function of several skeletal cell lineages. Independently of IGF-1, GH stimulates chondrocytes and osteoblasts; precursors of proliferation and differentiation. Simultaneously, bone resorption is mainly stimulated by IGF-1, whereas GH negatively modulates this effect by inducing the synthesis of osteoprotegerin by osteoblasts. Furthermore, GH may influence parathyroid hormone secretion pattern through phosphate retention, thus further influencing bone remodeling.

Children and adults with GH deficiency (GHD) often have decreased bone mineral density (BMD) and bone mineral content (BMC), as measured by dual energy x-ray absorptiometry [3-6]. Most likely, GHD severity influences the degree of bone loss. Several predictors of BMD response to $\mathrm{GH}$ replacement in adults have been described; baseline BMD, gender (men show better improvement than women in randomized and prospective studies), duration of treatment ( $>1$ year), concomitant pituitary deficiencies, and severity of GHD [7].

Fracture risk in patients with GHD is also high [8]; patients with hypopituitarism and GHD have a 2-5 times increased fracture risk, which is dependent on baseline disease and multiple hormonal replacements. Of note, BMD, as in other patients with pituitary tumors (acromegaly, for example) might not be a good predictor of fracture risk [9]. A long-term (6-year) study [10] revealed a higher

Maria Fleseriu

fleseriu@ohsu.edu

1 Northwest Pituitary Center, Department of Medicine, Division of Endocrinology, Diabetes and Metabolism, and Department of Neurological Surgery, Oregon Health \& Science University, Mail Code: CH8N, 3303 SW Bond Ave., Portland, OR 97239, USA number of radiographic vertebral fractures in untreated compared with treated GHD patients, irrespective of age. Interestingly, in a large prospective database study of GHD patients [11], those with osteoporosis had similar fracture rates either with or without $\mathrm{GH}$ replacement, but $\mathrm{GH}$ replacement prevented fractures only for patients without osteoporosis at baseline.

Despite BMD increase, the benefit of GH replacement on bone is not obvious in all GHD patients and an optimal $\mathrm{GH}$ dose to achieve the highest BMD increase remains unclear [8]; some studies suggest no correlation between $\mathrm{GH}$ dose and BMD change [9].

Over the last decade, GH has been US Food and Drug Administration approved for other adult indications, in addition to GHD, including human immunodeficiency virus associated cachexia and short bowel syndrome. Therefore, given an exponentially increasing number of patients living with osteoporosis, there is renewed interest in $\mathrm{GH}$ use to treat age-related bone loss.

Indeed, BMD in older women has been shown to be positively correlated with endogenous $\mathrm{GH}$ secretion and decreased IGF-1 has been associated with an increased risk of osteoporotic fractures [12]. Based on well-described GH actions on bone with improved BMD and expected IGF-1 decrease with age, many authors have hypothesized that $\mathrm{GH}$ administration could improve BMD in older osteoporotic adults, especially women, even in the absence of GHD per se.

Barake et al. [13], conducted a systematic literature review and a meta-analysis to answer an important question; is $\mathrm{GH}$ beneficial in patients with age-related bone loss, by both increasing BMD, but more importantly by decreasing fracture risk, even in the absence of pituitary deficiencies (and documented GHD).

Over the years, GH and osteoporosis research has been scarce, with conflicting results. Notably, Barake et al. [13] contacted the corresponding authors of three studies with inconsistent data and fortunately were able to obtain additional data on two [14, 15]. 
For their meta-analysis [13], data were limited to seven randomized studies that included 272 post-menopausal women (61-69 years), treated with either GH or control (6-24 months), and an eighth extension trial. Complicating the analysis was that all women received concurrent osteoporosis therapies in all, but one study. Also many women had been treated with estrogen, including oral, which may have rendered $\mathrm{GH}$ treatment ineffective; women taking oral estrogen require higher doses ( $30 \%$ higher) to achieve the same IGF-1 levels.

Barake et al. [13] combined results of three randomized trials and noted no significant difference with $\mathrm{GH}$ therapy on BMD (total hip, femoral neck or lumbar spine) or BMC when compared to placebo. Bone biomarkers increased, however the trend only reached statistical significance for carboxy-terminal propeptide of procollagen type I. It is not clear if the lack of a significant BMD change was related to the short duration of study GH therapy; patients were older and remodeling efficacy seems to decrease with aging. Conversely, based on four studies (short and long-term), a significant decrease in the risk of fracture, $(\mathrm{RR}=0.63[0.46,0.87])$ was observed overall with GH treatment. Notably, without the long-term study [16]. $\mathrm{GH}$ role in decreasing fracture risk would have been nonsignificant.

There is, however, a paucity of studies, that have addressed the effect of $\mathrm{GH}$ receptor exon 3 deletion polymorphism [7] in predicting skeletal fragility in hypopituitary adults with GHD and its potential implication on the response to $\mathrm{GH}$ replacement therapy. GHR polymorphism can partially explain the wide responses to $\mathrm{GH}$ in agerelated osteoporosis; any potential implication for pharmacogenetics needs further study.

The safety of GH treatment has been well described [9, 11]. Studies included by Barake et al. [13], observed previously recognized fluid retention side effects (peripheral edema, myalgias, arthralgias, and carpal tunnel syndrome), however, frequency and severity of events were not systematically reported. When described, symptoms were usually transient and mostly relieved by decreasing the $\mathrm{GH}$ dose.

Largely, all studies reviewed in Barake et al. [13], metaanalysis had significant design and follow-up caveats; these are well acknowledged. Overall, there were no major adverse events observed and mortality was not increased, but not all studies reported uniformly all side effects.

In conclusion, $\mathrm{GH}$ treatment in patients without documented GHD might indeed decrease fracture risk in some patients, especially in a subgroup of osteoporotic patients, with lower IGF-1 levels. Larger and long-term randomized studies are needed to elucidate the effects of a stable longterm regimen of GH treatment in both men and women with age-related osteoporosis on BMD, bone architecture and fracture risk either consecutive or concomitant to antiresorptive or anabolic treatment.

Currently, there is no evidence to recommend GH use for osteoporosis treatment and fracture prevention. Up-to-date prevention and treatment of osteoporosis guidelines in both men and women should be followed to decrease severe associated morbidity and mortality.

\section{Compliance with ethical standards}

Conflict of interest Dr. Maria Fleseriu has been a principal investigator with research support to Oregon Health \& Science University regarding clinical research studies with NovoNordisk and Pfizer, and she has been previously an occasional scientific consultant for Pfizer.

\section{References}

1. A. Giustina, G. Mazziotti, E. Canalis, Growth hormone, insulinlike growth factors, and the skeleton. Endocr. Rev. 29, 535-559 (2008)

2 E. Varlamov, M. Fleseriu, Growth hormone deficiency and replacement effect on adult bone mass: a clinical update. Curr. Opin. Endo. Metab. Res. In Press (2018)

3. G.I. Baroncelli, S. Bertelloni, C. Ceccarelli, G. Saggese, Measurement of volumetric bone mineral density accurately determines degree of lumbar undermineralization in children with growth hormone deficiency. J. Clin. Endocrinol. Metab. 83, 3150-3154 (1998)

4. A.M. Boot, M.A.M.J. Engels, G.J.M. Boerma, E.P. Krenning, S. M.P.F. de Muinck Keizer-Schrama, Changes in bone mineral density, body composition, and lipid metabolism during growth hormone $(\mathrm{GH})$ treatment in children with $\mathrm{GH}$ deficiency. J. Clin. Endocrinol. Metab. 82, 2423-2428 (1997)

5. S.J. Holmes, G. Economou, R.W. Whitehouse, J.E. Adams, S.M. Shalet, Reduced bone mineral density in patients with adult onset growth hormone deficiency. J. Clin. Endocrinol. Metab. 78, 669-674 (1994)

6. T. Rosen, T. Hansson, H. Granhed, J. Szucs, B.A. Bengtsson, Reduced bone mineral content in adult patients with growth hormone deficiency. Acta Endocrinol. 129, 201-206 (1993)

7. N.A. Tritos, A. Klibanski, in Chapter Nine-Effects of Growth Hormone on Bone, ed. by F.F Casanueva. Progress in Molecular Biology and Translational Science, vol 138 (Academic Press publications, London, UK, 2016), pp. 193-211

8. N.A. Tritos, Skeletal health in adult growth hormone deficiency. Endocrine 52, 1-2 (2016)

9. M. Barake, A. Klibanski, N.A. Tritos, Effects of recombinant human growth hormone therapy on bone mineral density in adults with growth hormone deficiency: a meta-analysis. J. Clin. Endocrinol. Metab. 99, 852-860 (2014)

10. G. Mazziotti, M. Doga, S. Frara, F. Maffezzoni, T. Porcelli, L. Cerri, R. Maroldi, A. Giustina, Incidence of morphometric vertebral fractures in adult patients with growth hormone deficiency. Endocrine 52, 103-110 (2016)

11. D. Mo, M. Fleseriu, R. Qi, N. Jia, C.J. Child, R. Bouillon, D.S. Hardin, Fracture risk in adult patients treated with growth hormone replacement therapy for growth hormone deficiency: a prospective observational cohort study. Lancet Diabetes Endocrinol. 3, 331-338 (2015)

12. C. Ohlsson, D. Mellstrom, D. Carlzon, E. Orwoll, O. Ljunggren, M.K. Karlsson, L. Vandenput, Older men with low serum IGF-1 
have an increased risk of incident fractures: the MrOS Sweden study. J. Bone Miner. Res. 26, 865-872 (2011)

13. M. Barake, A. Arabi, N. Nakhoul, G. El-Hajj Fuleihan, S. El Ghandour, A. Klibanski, N.A. Tritos, Effects of growth hormone therapy on bone density and fracture risk in age-related osteoporosis in the absence of growth hormone deficiency: a systematic review and meta-analysis. Endocrine. (2017) https://doi.org/10. 1007/s12020-017-1440-0

14. L. Holloway, L. Kohlmeier, K. Kent, R. Marcus, Skeletal effects of cyclic recombinant human growth hormone and salmon calcitonin in osteopenic postmenopausal women. J. Clin. Endocrinol. Metab. 82, 1111-1117 (1997)

15. K. Landin-Wilhelmsen, A. Nilsson, I. Bosaeus, B.A. Bengtsson, Growth hormone increases bone mineral content in postmenopausal osteoporosis: a randomized placebo-controlled trial. J. Bone Miner. Res. 18, 393-405 (2003)

16. E. Krantz, P. Trimpou, K. Landin-Wilhelmsen, Effect of growth hormone treatment on fractures and quality of life in postmenopausal osteoporosis: a 10-year follow-up study. J. Clin. Endocrinol. Metab. 100, 3251-3259 (2015) 